\title{
Modeling of species and charge transport in Li-Ion Batteries based on non-equilibrium thermodynamics
}

\author{
A. Latz, J. Zausch and O. Iliev \\ Fraunhofer Institut für Techno- und Wirtschaftsmathematik \\ Kaiserslautern, Germany
}

July 6, 2010

\begin{abstract}
In order to improve the design of Li ion batteries the complex interplay of various physical phenomena in the active particles of the electrodes and in the electrolyte has to be balanced. The separate transport phenomena in the electrolyte and in the active particle as well as their coupling due to the electrochemical reactions at the interfaces between the electrode particles and the electrolyte will influence the performance and the lifetime of a battery. Any modeling of the complex phenomena during the usage of a battery has therefore to be based on sound physical and chemical principles in order to allow for reliable predictions for the response of the battery to changing load conditions. We will present a modeling approach for the transport processes in the electrolyte and the electrodes based on non-equilibrium thermodynamics and transport theory. The assumption of local charge neutrality, which is known to be valid in concentrated electrolytes, is explicitly used to identify the independent thermodynamic variables and fluxes. The theory guarantees strictly positive entropy production. Differences to other theories will be discussed.
\end{abstract}

\section{Introduction}

Mathematical modeling of Li-ion batteries on cell level was pioneered by the work of Newman and his coworkers $[1,2,3]$ and extended and refined by many other authors $[4,5,6]$. The modeling approach is based on transport equations for Li ions and charges in the electrolyte as well as in the active particles of cathode and anode (for an illustration of the Lithium Ion battery see Fig. 1). Originally the electrodes were considered as porous media [1] made of a porous active particle skeleton filled with electrolyte. Later the porous model was derived with the help of volume averaging techniques for some set of equations for the different transport mechanisms in electrolyte and in the solid active particles [7]. The transport of charges and species between the electrolyte and the active particles was described with the help of a Butler - Volmer reaction model [2] and some assumptions about continuity conditions for charge and species flux. So far approaches where the active particles are resolved and the transport in particles and electrolytes are treated separately are rare $[8,9]$. But whether one starts directly with the porous electrode model or with a model resolving the fine structure of the electrode, it is in both cases important to base the battery model on thermodynamically consistent concepts, especially if at some point also the local heat production is to be simulated. 




Figure 1: Illustration of a Lithium ion battery. The spheres are the active particles of the porous electrodes in which Lithium Ions (green dots) can be stored. The voids between particles and between the electrodes are filled with an electrolyte in which Lithium ions diffuse and migrate. Electrons, on the other hand, have to move through the external circuit.

Charge transport mechanisms in active particles and in electrolyte are completely different on the microscopic (atomic) scale. In active particles, charge is transported mainly by pure electronic conduction. The contribution of the ion diffusion in the active particles to the electric current can be neglected due to the large mobility of the electrons compared to the ions. Charge transport in the electrolyte, on the other side, is exclusively due to ionic transport. In fact, the transfer of electrons into the electrolyte would result in the reduction of $\mathrm{Li}$ ions in the electrolyte to metallic Lithium, and is considered to be one of the many degradation mechanisms in Li ion batteries [10]. Due to the large mobility of electrons local charge neutrality is easily maintained in the active particles. The charge of an inserted Li Ions is instantaneously shielded by local rearrangements of electronic charges and the transport of electrons into the active particles over the current collectors. In the electrolyte the transport of species (ions) and charge is strongly coupled. Both, charge and species fluxes are caused by gradients in the chemical potential as well as by gradients in the electrical potentials. The constitutive relations for the charge and species fluxes describing these relations are well known for dilute electrolytes [2]. In batteries we have to deal with highly concentrated electrolytes. Some relations for these electrolytes are also derived in [2] combining multicomponent diffusion theory and considerations for chemical equilibria between reacting species in the electrolyte. As it will be shown below, the result is at variance with the general form of constitutive relations in ionic liquids, usually obtained in non equilibrium thermodynamics [11, 12]. A main contribution of our paper is therefore the careful rederivation of the constitutive relations for ion and charge flux in a mixture of a fully dissociated binary salt in a neutral solvent, using the well known concepts of nonequilibrium thermodynamics [11, 12]. As it is known that local charge neutrality is preserved in concentrated electrolytes except for the diffuse part of the double layer around active particles [2], we make explicit use of this property in our derivation of the constitutive relations. Due to the limited space for this article, details of the more general derivation, including thermal fluctuations will be given elsewhere [13]. With these equations the transition to the effective porous medium theory for cathode and anode can be obtained with standard techniques as e.g. volume averaging [7]. 


\section{Model}

The starting point for a continuum model of charge and species transport in a Li-ion battery are the conservation equation for Li-ion concentration $c$ and charges $q$. The continuity equation for the concentration of Li Ions $c$ is given by

$$
\frac{\partial c}{\partial t}=-\vec{\nabla} \vec{N}_{+}
$$

Here $\vec{N}_{+}$is the flux of Li ions. The equation for the charge concentration is given by

$$
\frac{\partial q}{\partial t}=-\vec{\nabla} \vec{j}
$$

where $\vec{j}$ is the electrical current. The approximation of charge neutrality requires not only that the time derivative in (2) is identical zero, but that the local charge $q$ vanishes i.e. $q \equiv 0$. The main challenge for a constitutive theory is to derive a thermodynamically consistent relation for the fluxes $\vec{N}_{+}$and the electrical current $\vec{j}$. Also, the influence of solvent molecules and negative ions to the transport properties has to be clarified.

\subsection{Charge and species transport in a concentrated electrolyte}

To obtain a thermodynamically consistent model for charge and ion fluxes in the electrolyte we apply the well known formalism of non-equilibrium thermodynamics $[11,12]$ to a mixture of fully dissociated binary salt and a solvent. The concentrations of positive and negative ions with charge $z_{+}$and $z_{-}$are $c_{+}$and $c_{-}$, respectively. The concentration of the solvent is $c_{0}$. Instead of motivating our theory with considerations from dilute electrolyte theory, we are considering the opposite limit of concentrated electrolytes. In this limit the Debye length $\lambda_{D}$ is so small, that local charge fluctuations are restricted to scales well below about $100 \mathrm{~nm}$ [2]. We therefore impose local charge neutrality $z_{+} c_{+}+z_{-} c_{-}=0$ in our derivation exactly. This will allow us to identify the relevant measurable transport coefficients for the electrolytes used in $\mathrm{Li}$ ion batteries. For example, the strong Coulomb interaction between the ions prevent independent motion of ions to occur on the scale of battery cell dimensions. The main diffusion process will be correlated interdiffusion with a uniquely defined interdiffusion coefficient for positive and negative ions. Independent self diffusion of the different ions with different self diffusion coefficient leading to slow charge separation is excluded in a strictly charge neutral system.

Under normal operation conditions for a Li ion battery we may safely assume that convection can be excluded as transport mechanism. This assumption allows to eliminate the concentration of the neutral solvent as independent variable. With $M_{0}, M_{+}, M_{-}$being the molar masses of solvent and positive and negative ions respectively we get in the absence of convection the relations

$$
M_{0} d c_{0}+M_{+} d c_{+}+M_{-} d c_{-}=0
$$

for the changes in the respective concentrations. Charge neutrality is then used to eliminate the concentration of the negative ions using the relation

$$
z_{+} c_{+}+z_{-} c_{-}=0
$$

It is therefore sufficient to determine the transport equations for the concentration $c=c_{+}=-\frac{z_{-}}{z_{+}} c_{-}$. Using the constraints between changes in energy density $u$, entropy density $s$, concentration $c$ and charge 
density $q$, and denoting as usually the temperature by $T$, the thermodynamic relation for the electrolyte in an external field $\Phi$ can be written as

$$
d u=T d s+\mu d c+\Phi d q
$$

Due to the imposed charge neutrality the changes in the charge are zero i.e. $d q=0$. The energy density also contains the contribution from the electric fields [14]. The effective chemical potential $\mu$ is a combination of the chemical potentials $\mu_{+}, \mu_{-}$and $\mu_{0}$ of the ions and the solvent.

$$
\begin{aligned}
\mu & =\tilde{\mu}_{+}-\frac{z_{+}}{z_{-}} \tilde{\mu}_{-} \\
\tilde{\mu}_{+} & =\mu_{+}-\frac{M_{+}}{M_{0}} \mu_{0} \\
\tilde{\mu}_{-} & =\mu_{-}-\frac{M_{-}}{M_{0}} \mu_{0}
\end{aligned}
$$

Formally, the chemical potential $\mu$ is the work to be performed for injecting $1 \mathrm{~mol}$ of Li ions from infinity in the electrolyte including the work to rearrange the negative ions and neutral solvent molecules such that charge neutrality and momentum are conserved. The entropy production $\sigma$ in the system fulfills the relation $[12,13]$

$$
T d \sigma=-\overrightarrow{J_{s}} \vec{\nabla} T-\vec{N}_{+} \vec{\nabla} \tilde{\mu}_{+}-\vec{N}_{-} \vec{\nabla} \tilde{\mu}_{-}-\vec{j} \vec{\nabla} \Phi
$$

The electric current is given by

$$
\vec{j}=z_{+} \vec{N}_{+}+z_{-} \vec{N}_{-}
$$

Using this relation to eliminate the flux of negative ions $\vec{N}_{-}$, we obtain

$$
T d \sigma=-\overrightarrow{J_{s}} \vec{\nabla} T-\vec{N}_{+} \vec{\nabla} \mu-\vec{j} \vec{\nabla}\left(\Phi+\frac{\tilde{\mu}_{-}}{z_{-} F}\right)
$$

where $\mathrm{F}$ is the Faraday number. Note that the form of the entropy production determines the set of independent thermodynamic forces and thus the correct form of the Onsager relations in the constitutive equations for the fluxes [12]. In the following we neglect for simplicity thermal fluctuations e.g. $d T=0$.

Having identified the independent thermodynamic variables and forces, it is possible to formulate the constitutive relations for the fluxes. Under the necessary requirement of strictly positive entropy production they have the general form

$$
\begin{aligned}
\vec{N}_{+} & =-L_{11} \vec{\nabla} \tilde{\mu}_{+}-L_{12} \vec{\nabla} \tilde{\Phi} \\
\vec{j} & =-L_{21} \vec{\nabla} \tilde{\mu}_{+}-L_{22} \vec{\nabla} \tilde{\Phi}
\end{aligned}
$$

where $\tilde{\Phi}=\Phi+\frac{\tilde{\mu}_{-}}{z_{-F}}$. $\tilde{\Phi}$ may be interpreted as the renormalized effective potential due to the partial shielding of the external potential by the negative ions. The Onsager matrix $L_{i j}$ has to be symmetric positive definite i.e. $L_{12}=L_{21}$. A simple rearrangement of (12), (13) and introduction of standard notation leads to

$$
\begin{aligned}
\vec{N}_{+} & =-D_{e} \vec{\nabla} c+\frac{t_{+}}{F z_{+}} \vec{j} \\
\vec{j} & =-\kappa \vec{\nabla} \tilde{\Phi}-\kappa \frac{t_{+}}{F z_{+}}\left(\frac{\partial \tilde{\mu}_{+}}{\partial c}\right) \vec{\nabla} c
\end{aligned}
$$


The transport coefficients $D_{e}, t_{+}, \kappa$ are the ion collective interdiffusion coefficient of the fully interacting system at zero electric current, the transference number and the ion conductivity, respectively. They are given by

$$
\begin{aligned}
\kappa & =L_{22} \\
t_{+} & =\frac{z_{+} L_{12} F}{\kappa} \\
D_{e} & =\left(L_{11}-\kappa \frac{t_{+}^{2}}{F^{2} z_{+}^{2}}\right)\left(\frac{\partial \tilde{\mu}_{+}}{\partial c}\right)=\frac{\operatorname{det} L}{L_{22}}\left(\frac{\partial \tilde{\mu}_{+}}{\partial c}\right)
\end{aligned}
$$

The constitutive relation for the negative ion flux is a consequence of the definition of the current (10) and charge neutrality

$$
\vec{N}_{-}=-D_{e} \vec{\nabla} c_{-}+\frac{t_{-}}{F z_{-}} \vec{j}
$$

Here $t_{-}=1-t_{+}$is the transference number of negative ions. The interdiffusion coefficient for the density of negative ions is the same as the one for the positive ion density due to the imposed charge neutrality. This result is consistent with fundamental Green Kubo relation for the interdiffusion coefficient in a binary systems [15]. For comparison with experiments it is important to realize that it is the interdiffusion coefficient, and not the self diffusion coefficients, which has to be determined in order to simulate the behavior of Li ion batteries. In general the two self diffusion coefficients and the interdiffusion coefficient are mutually different from each other $[15,16]$.

It is also important to note that the relation (15) is different from the one derived in [2]. The constitutive relation for the electrical current in [2] depends on the type of chemical reactions in the electrolyte and is not just a property of the local gradients in the independent field variables. This ansatz causes an asymmetry in the relations for the ion flux and the electrical current, which violates the fundamental Onsager relation necessary for strictly positive entropy production. In the case of a simple ion insertion reaction at the electrodes the factor $t_{+}$in the relation for the current in (15), in [2] is replaced by $-\left(1-t_{+}\right)$. I.e. the absolute value and the sign in front of the $\vec{\nabla} c$ term are different compared to our theory. The isothermal entropy production for the two models are

$$
T \sigma_{N}=D_{e}\left(\frac{\partial \mu}{\partial c}\right)_{T}(\vec{\nabla} c)^{2}+\frac{\vec{j}^{2}}{\kappa}-\left(\frac{\partial \mu}{\partial c}\right)_{T} \frac{\vec{j} \vec{\nabla} c}{F}
$$

in the theory of $[2]$ and

$$
T \sigma_{L Z}=D_{e}\left(\frac{\partial \mu}{\partial c}\right)_{T}(\vec{\nabla} c)^{2}+\frac{\vec{j}^{2}}{\kappa}
$$

in our case. Since the thermodynamic derivative $\left(\frac{\partial \mu}{\partial c}\right)_{T}$ and the interdiffusion coefficient $D_{e}$ are always positive the model presented here leads as expected to the strictly positive entropy production in Eq. (21). The last term in Eq. (20) does not have a definite sign and therefore allows in principal for negative entropy production.

Since the relation used in [2] is used as starting point for many battery modeling approaches [17, 3, 8], differences to our approach may be expected (cf Ref. [18]). 


\subsection{Transport in active particles}

For the transport in the active particles, the diffusion and the conduction are essentially decoupled, since the mobility of the ions is much smaller than the one of the electrons and therefore the electric conduction is nearly completely carried by the electrons. The ions in the active particles are transported by diffusion only. The constitutive relations for ion flux and electrical current are given by

$$
\begin{aligned}
\vec{N}_{+} & =-D_{s} \vec{\nabla} c \\
\vec{j} & =-\sigma_{s} \vec{\nabla} \Phi
\end{aligned}
$$

where $\sigma_{s}$ and $D_{s}$ are the electronic conductivity and the ion diffusion coefficient respectively. As long as the binder and the additives in the electrodes are not treated as different phases the electronic conductivity is an effective conductivity of active particles and additives.

\subsection{Intercalation modeling and interface conditions}

For the coupling of the transport in the active particles and in the solid electrolyte, interface conditions have to be formulated. The interface conditions describe the intercalation reaction and the deintercalation reaction respectively on the mesoscopic scale (i.e beyond the scale of the diffuse layer [2]). It is assumed that the transport of ions across the interface is completely described by the Butler Volmer expression $i_{s e}$ for the intercalation reaction [2].

$$
i_{s e}=i_{0}\left(\exp \left[\frac{\alpha_{a} F}{R T} \eta_{s}\right]-\exp \left[\frac{-\alpha_{c} F}{R T} \eta_{s}\right]\right)
$$

$\alpha_{A}$ and $\alpha_{C}$ with $\alpha_{A}+\alpha_{C}=1$ are weighting the anodic and the cathodic contribution of the overpotential $\eta_{s}$ to the overall reaction. The overpotential is defined by

$$
\eta_{s}:=\Phi_{s}-\Phi_{e}-U_{0}
$$

Here $U_{0}$ is the half cell open circuit potential of the respective electrode.

The amplitude $i_{0}$ is given by

$$
i_{0}=k(c)^{\alpha_{a}}\left(c_{s}\right)^{\alpha_{a}}\left(1-\frac{c_{s}}{c_{s, \max }}\right)^{\alpha_{c}}
$$

$k$ is a reaction rate. $c_{s, \max }$ is the maximum concentration which can be stored in the active particle. We assume that $\mathrm{Li}$ ions are not stored in the double layer (i.e. all Li ions are intercalated in the active particle or released into the electrolyte). There should also be no flux of negative charges across the double layer. Neither enter electrons the electrolyte nor intercalate negative ions from the electrolyte in the active particles under ideal conditions. This especially means that the total current across the electrolyte-particle interface is due to the transport of positive ions only. If the particle is completely filled

i.e. $c=c_{s, \text { max }}$, it has to be made sure by the interface conditions that no electrical current $\vec{j}$ is carried by negative charge carriers across the interface. These conditions can be formulated mathematically in 
the following way with the normal $\vec{n}$ pointing from the solid into the electrolyte

$$
\begin{aligned}
\vec{j}_{s} \vec{n} & =\vec{j}_{e} \vec{n} \\
\vec{j}_{s} \vec{n} & =i_{s e} \\
\vec{N}_{+, s} \vec{n} & =\vec{N}_{+, e} \vec{n} \\
\vec{N}_{+, s} \vec{n} & =\frac{i_{s e}}{F}
\end{aligned}
$$

To solve the model for the battery problem additional boundary conditions have to be provided for the potential and the current at the current collectors in contact with the active particles. These conditions are determined by the operating conditions of the battery. In addition the ion fluxes have to be set to zero at all external boundaries.

\section{Conclusions}

We derived a thermodynamically consistent model for transport of charges in a battery cell, consisting of active particles and electrolyte in cathode and anode. The modeling of the separator was not addressed, but it is straightforward using effective diffusion coefficients and ionic conductivities in the electrolyte theory, if the separator itself is a porous structure [1].To test the model, a 1-D porous electrode version of the model was implemented in the commercial software package Comsol and compared with the model used in [1]. Detailed results will be presented in [18]. Numerical algorithm for the introduced here model, as well as its numerical study for 3D geometry, are presented in [19]

Acknowledgment. The work was supported by the Fraunhofer system research for electromobility (FSEM) within the economic stimulus package II of the German Ministry of Education and Research.

\section{References}

[1] Fuller, T.F., Doyle, M., Newman, J.: Simulation and optimization of the dual lithium ion insertion cell. J. Electrochem. Soc. 141 (1994) 1-10

[2] Newman, J., Thomas-Alyea, K.E.: Electrochemical Systems. Wiley (2004)

[3] Thomas, K.E., Newman, J., Darling, R.M.: Mathematical modeling of lithium batteries. In Schalkwijk, W.A., Scrosati, B., eds.: Advances in Lithium-Ion Batteries. Kluver (2002) 345-392

[4] Botte, G.G., Subramanian, V.R., White, R.E.: Mathematical modeling of secondary lithium batteries. Electrochimica Acta 45 (2000) 2595-2609

[5] Danilov, D., and, P.H.L.N.: Mathematical modelling of ionic transport in the electrolyte of li-ion batteries. Electrochimica Acta 53 (2008) 5569-5578

[6] Olesen, L.H., Bazant, M.Z., Bruus, H.: Strongly nonlinear dynamics of electrolytes in large ac voltages. arXiv:0908.3501 (2009)

[7] Wang, C.Y., Gu, W.B., Liaw, B.Y.: Micro-macroscopic coupled modeling of batteries and fuel cells. i. model development. J. Electrochem. Soc. 145 (1998) 3407-3417 
[8] Wang, C.W., Sastry, A.M.: Mesoscale modeling of li-ion polymer cell. J. Electrochem. Soc. 154 (2007) A1035-A1047

[9] Zausch, J., Latz, A., Schmidt, S., Less, G.B., Seo, J.H., Han, S., Sastry, A.M.: Micro-scale modelingof li-ion batteries, parameterization and validation. to be published (2010)

[10] Vetter, J., Novak, P., Wagner, M.R., Veit, C., Möller, K.C., Besenhard, J.O., Winter, M., WohlfahrtMehrens, M., Vogler, C., Hammouche, A.: Ageing mechanisms in lithium-ion batteries. J. Pow. Sources 147 (2005) 269-281

[11] Landau, L.D., Lifshitz, E.M.: Electrodynamics of Continous Media. Pergamon, Oxford (1984)

[12] de Groot, S., Mazur, P.: Non-Equilibrium Thermodynamics. Dover (1984)

[13] Latz, A., Zausch, J.: Derivation of a thermodynamic consistent mesoscopic battery model. to be published (2010)

[14] Liu, M.: Hydrodynamic theory of electromagnetic fields in continous media. Phys.Rev.Lett. 70 (1993) 3580-3583

[15] Hansen, J.P., McDonald, I.R.: Theory of Simple Liquids. Academic Press (1986)

[16] Aouizerat-Elarby, A., Dez, H., Prevel, B., Jal, J., Bert, J., Dupuy-Philon, J.: Diffusion processes in LiCl, R H2O solutions. Journal of Molecular Liquids 84(3) (2000) 289-299

[17] Doyle, M., Newman, J., Gozdz, A.S., Schmutz, C.N., Tarascon, J.M.: Comparison of modeling predictions with experimental data from plastic lithium ion cells. J. Electrochem. Soc. 143 (1996) 1890-1903

[18] Latz, A., Zausch, J.: Mesoscopic modeling and simulation of charge and ion transport in li ion battery cells. In: Proceedings Dechema Conference on Materials for Energy. (2010)

[19] Popov, P., Vutov, Y., S.Margenov, O.Iliev: Finite volume discretization of nonlinear diffusion in li-ion batteries. In N.Kolkovska, et al., eds.: Numerical Methods and Applications 2010. Springer (2011) ... 